\title{
Les français du Canada : faits linguistiques, faits de langue
}

Gisèle Chevalier

CRLA, Université de Moncton

La traduction d'ouvrages littéraires rédigés dans des langues régionales soulève le problème d'accès à des ouvrages de référence fiables décrivant leurs propriétés. Des dictionnaires, des glossaires, des brochures répertorient les aspects lexicaux des parlers régionaux. Non seulement sont-ils peu nombreux et fragmentaires, mais aussi, et surtout, ils ne représentent pas toujours la langue dans sa modernité ${ }^{1}$. Par ailleurs, il n'existe à peu près pas de grammaire qui décrive les parlers régionaux. Les descriptions qui ont été faites de la morphologie et de la syntaxe des français d'Amérique sont éparpillées dans des revues linguistiques. Ces travaux sont difficiles à colliger, ils ne sont pas toujours faciles à aborder et il faut mettre un certain temps pour en extraire les informations qui nous sont pertinentes.

Nous n'avons certainement pas la prétention de combler cette lacune en quelques pages. Nous nous proposons toutefois de présenter un inventaire des particularités morphosyntaxiques qui caractérisent les variétés de français au Canada en les situant les unes par rapport aux autres à l'intérieur d'un cadre conceptuel qui fait appel à la trajectoire des parlers de France qui se sont implantés en Amérique du nord et à la théorie du contact linguistique, que nous présenterons dans la première partie de l'article.

\section{Le contexte : Les souches de français au Canada}

Les variétés de français canadiennes se divisent en deux grands groupes : d'une part, les variétés acadiennes implantées dans les provinces maritimes à partir de 1604 et, d'autre part, les variétés laurentiennes qui se sont développées dans la vallée du Saint-Laurent après la fondation de Québec en 1608. Les Français qui ont colonisé l'Amérique au XVII ${ }^{\text {e }}$ siècle venaient de l'ouest de la France. Ils parlaient le dialecte de leurs régions respectives. Plusieurs avaient été exposés au français de l'Île-de-France, même s'ils ne le parlaient pas couramment. Environ la moitié était alphabétisée.

\footnotetext{
${ }^{1}$ Le dictionnaire $d u$ français acadien d'Yves Cormier (1999) est à ce jour ce qu'il y a de plus complet pour le vocabulaire.
} 
Les variétés acadiennes et laurentiennes se distinguent les unes des autres par la provenance des colons. Ceux qui se sont établis dans les provinces maritimes provenaient essentiellement de deux régions dites "patoisantes", celles du Poitou et de la Saintonge, alors que les vagues d'émigration ultérieures, qui se dirigeaient vers le Québec, amenaient des colons de provenance plus diversifiée. Un nombre important de dialectes en sont venus à se côtoyer. Ils n'étaient pas toujours inter-compréhensibles, ce qui a favorisé une certaine homogénéisation en faveur du français émergeant en France à l'époque de la colonisation (Poirier 1978; Mougeon et Beniak 1989)2. Les aventuriers et les découvreurs qui ont colonisé par la suite l'Ontario et l'ouest canadien et américain étaient pour la plupart originaires du Québec ou avaient transité par le Québec. C'est donc la koïnè de la vallée du Saint-Laurent qui s'est propagée.

À ce jour, les variétés de l'ouest ne se démarquent guère du français québécois que par les traits suprasegmentaux (la prosodie, soit le rythme, l'accent, l'intonation) et par le degré d'anglicisation du lexique (Mougeon et Beniak 1989 : 1-14; Mougeon 2005). Le français de souche acadienne se démarque du français laurentien par son hétérogénéité sur le plan régional et par un riche héritage encore vivant d'éléments des vieux parlers. En effet, l'isolement et l'interruption précoce de l'émigration vers l'Acadie des maritimes ont amplifié le conservatisme inhérent aux langues transplantées.

\section{Cadre conceptuel}

\subsection{Le français de référence}

Parler des particularismes d'une variété linguistique présuppose l'existence d'une langue de référence. Bien qu'il ait perdu sa suprématie en tant que norme unique et irrémédiable, le français hexagonal contemporain (ou l'idée que l'on s'en fait) conserve son prestige ou son autorité dans notre (in)conscient linguistique. Le français québécois, à la faveur de son statut de langue officielle et majoritaire, a affirmé son identité durant le dernier tiers du $X X^{\mathrm{e}}$ siècle et il est devenu, pour certains aspects, plus innovateur que la langue «mère ». De plus, il ne subit qu'un contact faible avec l'anglais. Les minorités francophones du reste du Canada ressentent un tiraillement entre les normes identitaires, celles de la langue mère et celles de la langue sœur, et sont à la recherche de leur propre norme.

\footnotetext{
${ }^{2}$ Voir aussi le survol historique récent de Baronian (2006) qui retrace les établissements francophones sur l'ensemble de l'Amérique du Nord (Canada et États-Unis).
} 
Les faits de langue présentés dans les pages qui suivent seront pour cette raison examinés dans une triple perspective: celle de l'héritage français (les formes traditionnelles contre les formes normalisées ou innovatrices), celle des changements intrasystémiques (qui sont motivés par le système linguistique du français) et celle des changements intersystémiques (résultat du contact avec un nouveau système, en l'occurrence, l'anglais ${ }^{3}$ ). Nous ajouterons à l'occasion quelques pistes de réflexion sur les facteurs extralinguistiques (sociaux, personnels et psycholinguistiques) qui en motivent l'éclosion.

\subsection{Effets du contact avec l'anglais sur les variétés de français au Canada}

L'intensité du contact avec l'anglais (usage occasionnel, fréquent, quotidien) et le degré d'exposition au français (restreint ou étendu à différentes sphères d'activités sociales, professionnelles et grégaires) ont une influence plus ou moins importante sur le développement des répertoires et des usages linguistiques des locuteurs francophones du Canada. Dans la préface de leur collectif sur les français hors Québec, Raymond Mougeon et Édouard Beniak (1989 : 3-14) regroupent en trois processus distincts les effets du contact qu'ils ont observés dans le corpus franco-ontarien :

1) le transfert, qui regroupe : a) l'emprunt lexical : la télé => la t.v.; b) le calque : chercher quelque chose $\Rightarrow$ chercher pour ( to look for »), voir à la télévision => regarder sur la t.v. (« to watch on the t.v. ») ${ }^{4}$, et pour finir c) le transfert linguistique, soit l'aller-retour d'un code à l'autre au cours de la conversation (voir à la section 7.3);

2) les restructurations: par exemple, le nivellement des formes irrégulières des verbes : s'asseoir $\Rightarrow>$ s'assir, vous dites $\Rightarrow$ vous disez, l'emploi de l'indicatif au lieu du subjonctif : faut que je parte $\Rightarrow$ faut que je pars, le remplacement du pronom clitique me par la forme forte postposée au verbe : il me l'a dit => il l'a dit à moi; et, pour finir,

3) la réduction stylistique, soit la réduction ou l'abandon de variantes disponibles dans le français de référence, par exemple, perte de la distinction tu/vous en franco-terre-neuvien ou la disparition de la variante usuelle chez nous à côté de à la maison.

Les calques, les restructurations linguistiques et la réduction stylistique sont des phénomènes qui ne transgressent pas les règles du français, l'anglais

\footnotetext{
${ }^{3} \mathrm{Au}$ Manitoba et en Saskatchewan, le contact étroit entre le français et les langues amérindiennes a donné naissance a une langue mixte, le métchif, qui a à son tour subi une influence sensible de l'anglais (Papen 2006).

${ }^{4}$ En acadien on surveille la t.v. ('to watch the t.v.').
} 
n'apporte pas d'éléments linguistiques nouveaux, mais il agit comme catalyseur ou accélérateur du changement. La réduction des expressions (la simplification, alinéa 3, ci-haut) se produit davantage dans un milieu où l'accès à la langue est restreint.

\subsection{Parti-pris méthodologique : la linguistique de corpus}

Les traits linguistiques que nous allons discuter reposent sur des faits de langue observés dans des corpus oraux. Ces corpus sont des recueils d'entrevues ou de conversations enregistrées puis transcrites orthographiquement. Ils servent, en linguistique, à étudier les usages effectifs de la langue dans différents types de communautés. Nous privilégions les corpus oraux sociolinguistiques, c'est-à-dire ceux dont les chercheurs ont contrôlé les conditions de production (spontanées, semi-dirigées, formelles, avec ou sans présence d'observateur, appartenant ou non à la communauté) de même que les facteurs sociolinguistiques tels l'âge, le sexe, la scolarité, la classe socio-économique et, de plus en plus, les réseaux sociaux (ouverts ou fermés). Ils permettent de mettre les usages linguistiques en relation avec les facteurs extralinguistiques qui en favorisent l'emploi.

Grâce à la linguistique de corpus et au développement de logiciels adaptés au traitement linguistique de corpus oraux, il devient possible de dresser un inventaire des usages linguistiques contemporains fondé sur des productions authentiques. Une équipe de chercheurs mise sur pied par Sylvia Kasparian de l'Université de Moncton a œuvré à développer les ressources lexicales, morphologiques et syntactico-sémantiques d'un module acadien qui est maintenant incorporé au logiciel de traitement du langage NooJ (Silberztein $2008^{5}$ ). La démarche a consisté à appliquer les ressources linguistiques du module français de NooJ à une grande variété de corpus acadiens afin de départager les formes françaises des formes non reconnues par les dictionnaires français. Chaque forme légitime parmi les formes non reconnues est décrite dans une entrée du dictionnaire acadien de mots ou d'expressions du point de vue de ses propriétés grammaticales, morphologiques et syntactico-sémantiques. Il est également possible de lui attribuer des "gloses » en français de référence et en anglais (ou d'autres langues), de même que les marques d'emploi et les propriétés sociolinguistiques. Des grammaires morphologiques ont été développées pour rendre compte des règles d'accord et de conjugaison verbale idiosyncratiques, ainsi que des trucages orthographiques (Chevalier, Kasparian et Silberztein 2004).

\footnotetext{
${ }^{5}$ Le logiciel et le module acadiens peuvent être téléchargés gratuitement à l'adresse www.noojhlp.com.
} 
Le module acadien de NooJ consigne par conséquent les résultats des recherches sur les parlers acadiens, et il ouvre la possibilité de fournir des réponses fondées sur un bon bagage de faits empiriques aux questions que nous posent le public ou des groupes cibles, tels les traducteurs. Enfin, l'outil pourra servir à dresser dans des délais raisonnables, la nomenclature bilingue ou multilingue circonstanciée d'œuvres documentaires ou littéraires entières rédigées en français périphérique.

\section{3. Étude de cas}

Dans les pages suivantes, nous examinerons les faits de langue qui se présentent dans six échantillons de productions orales tirés de corpus acadiens du Nouveau-Brunswick ${ }^{6}$. L'échantillonnage n'est certainement pas représentatif, mais nous avons pris soin de concocter un mélange de générations, de régions et de mises en scène. Les extraits servent de point de départ à la discussion des particularismes parfois considérés comme typiques de telle ou telle variété. Nous situerons les faits acadiens observés par rapport aux variétés laurentiennes et hexagonales à l'intérieur du cadre conceptuel que nous venons d'esquisser, soit l'héritage des vieux parlers (point 4), les faits oraux (point 5), les changements suivant des tendances intra et inter-linguistiques (points 6 et 7$)^{7}$.

\section{1. Échantillons de productions en acadien du Nouveau-Brunswick ${ }^{8}$}

A. Une fillette de 9 ans de la Baie-Sainte-Anne (sud-est du N.-B.) restitue un conte de fée qui vient de lui être lu (Corpus de la Baie-Sainte-Anne, Chevalier 2006) :

Pis i ont vu un arbre pis l'arbre s'a ouvri pis la fille a marché dans l'arbre pis i a fermé pis là la fille i a donné un... / pis là Tom l'a ouvri pis la fille i a donné un wish //(un voeu) pis Tom a whishé que i savait touT quoi-ce que les fourmis faisaient dans leur nid pis les poissons faisaient dans l'eau

\footnotetext{
${ }^{6}$ Nous laissons aux spécialistes le soin de parler des variétés de la Nouvelle-Écosse (Flikeid 1989a/b), de l'Île du Prince-Édouard (King 2000) et de Terre-Neuve (Brasseur 2001).

${ }^{7}$ Hallion Bres (2006) présente une compilation des études menées sur les variétés ontariennes et de l'ouest dont nous nous inspirons.

${ }^{8}$ Il est d'usage de ne pas ponctuer les phrases dans les transcriptions de productions orales. La barre oblique marque les pauses et les hésitations, l'apostrophe indique l'élision de consonnes (contac'), la majuscule la prononciation non prévue d'une consonne (touT, iciT). Les italiques mettent en évidence les emprunts lexicaux dans les extraits et, dans le texte, ils signalent les exemples et mots en mention.
} 
B. Une adolescente de 14 ans de la ville de Dieppe (sud-est) s'entretient avec une camarade de classe à propos de sujets divers (Corpus AnnaMalenfant, Chevalier et Gauvin 1994) :

je pense que / i est important de ben élever ses enfants parce que sinon $i$ peuvent finir comme / comme s'être des bum pis I ça je veux pas que mes enfants turnont out de même

C. Deuxième adolescente de 14 ans du même corpus :

... demander de l'argent à mes parents c'est pas de problèmes pantoute je vas juste awoir cinq piasses i vont me le donner / but i faut je travaille pour / euh: /je gagne mon argent à babysitter mes fréres pis babysitter les p'tits brats qui restent par che nous pis / je l'utilise hum / euh che pas pour acheter des jeans ou juste pour aller aux movies whatever

Les locuteurs des extraits D, E et F sont de jeunes cadres professionnels interviewés sur le lieu de travail dans un contexte formel (Corpus Péronnet-Kasparian, Péronnet et Kasparian 1995) :

D. Cadre d'Edmundston (nord-ouest) :

alors on / on va voir pour des octrois aux autres paliers $\mathrm{d}^{\prime}$ gouvernement / euh / des octrois extérieurs même avec le domaine du privé / parce que là c'est / c'est un aut' chose qui s'en vient à la mode des / des / des / on appelle ça en anglais des joint ventures avec le private euh and public partnership alors ça on Eregarde à ça

E. Cadre de Bathurst (nord) :

pis aussi vu que chus surveillante euh / je remplace les personnes qu allent de l'hôpital pour la / soirée / pour la nuit disons / dépendant les shif' que je fais / ça-fait-que / c'est pour ça que je fais un petit peu de tout là / euh / les gens / si i ont des problèmes / n'importe quel problème qu'i vont avoir / c'est moi qu'i vont appeler en premier / pis moi euh / je fais d'aut' contac' / j'appelle d'aut' gens / c'est pas moi qui va changer la / la lumière / pis euh / arranger la toilette là mais je fais sûr que c'est faiT / euh

F. Cadre de Moncton (sud-est) :

le personnel ici est vraiment extraordinaire / i se dévouent beaucoup / i s'impliquent beaucoup dans les comités / euh / si que/ euh / y a pas grand chose que je ieux demande qu'i/ qu' i m'aideront pas avec là

\section{Faits typiquement acadiens, reliquats des parlers de la vieille France}

\subsection{Le lexique du vieil acadien}


La part la plus importante de traces des vieux parlers de France se trouve dans le vocabulaire: les vêtements sont encore désignés comme des hardes, espère-moi signifie attendre (quelqu'un). On a mal à l'échine (dos) ou on a reçu un coup sur le noucle de la queue (соссух), on grée un arbre de Noël, quelqu'un est bien gréé (bien habillé, bien mis), on amarre ses lacets. Il est paradoxal de qualifier ces expressions d'archaïsmes, suivant une certaine tradition philologique, alors qu'elles sont encore vivantes dans les parlers à l'étude, et parfois productives ${ }^{9}$.

\subsection{Les flexions verbales}

La terminaison $-i$ du participe passé de ouvrir $\left(\mathrm{A}^{10}\right.$ : l'arbre s'a ouvri; Tom l'a ouvri) et la finale -ont de la troisième personne du pluriel, affixée même aux verbes anglais (B : turnont out) sont les seules formes verbales dans ces extraits qui sont encore d'usage courant en acadien et non pas dans les autres variétés de français. La forme -ont est d'ailleurs en récession dans le nord-est du N.-B., où elle ne s'utilise que si le verbe a pour sujet le pronom ils et ce, uniquement par des locuteurs adultes à réseau social fermé (Beaulieu et Cichocki 2005). Dans le sudest, l'usage semble plus courant et il n'est pas régi par la contrainte du pronom : j'attends que mes partenaires arriviont pour m'aider (entendu en octobre 2007 à Moncton). Le participe passé en $-i$ comme dans fini s'étend à plusieurs verbes du troisième groupe : j'ai li, il a mourri, j'ai vi (vécu).

\subsection{Prépositions et conjonctions}

Comme en ancien français, l'acadien actuel utilise la préposition de avec les verbes modaux: aimer de, souhaiter de, espérer de, ainsi de suite (Péronnet et Kasparian 2002, 2004) ainsi que les conjonctions de subordination complexes (E : si que) ou encore quand que, comme que, comment que, combien que, comme dans les conjonctions figées en français contemporain parce que, lorsque, malgré que, pendant que... (Beaulieu 1996; Hallion Bres 2006). Quoique si que et quand que s'entendent également dans les variétés laurentiennes et en français hexagonal populaire, nous ne pouvons nous prononcer sur l'ampleur du phénomène que dans l'acadien du Nouveau-Brunswick.

\section{Faits d'oralité}

\subsection{Le discours en construction}

${ }^{9}$ De nos jours, un homme ou une femme se fait amarrer « pour éviter la famille »... (Chevalier et coll. 2004, $\underline{\text { www.cafnb.ca }}>>$ ressources et aide en français $>>$ parlers régionaux $\gg>$ glossaire acadien de la santé).

${ }^{10}$ Les majuscules A, B, C, etc. renvoient à l'échantillon correspondant de la section 3.1. Lorsque les exemples ne sont pas attestés dans les échantillons, nous indiquons le nom du corpus cité. 
Mis à part les faits qui précèdent et les effets du contact à l'anglais, le reste des faits linguistiques qui attirent notre attention dans les extraits acadiens sont en partage avec les variétés laurentiennes et hexagonales. Plusieurs sont attribuables aux spécificités de l'oral soit à son caractère : a) sonore (voir 5.2. Prononciation); b) interactif (les marques d'interaction et le système de "ponctuation » orale comme chez $\mathrm{A}:$ pis répété à chaque étape du récit; $\mathrm{B}:$ ben, $\mathrm{D}$ : alors, F : pis, ça fait que, là, pis moi, pis euh; et c) spontané, non planifié, ce que signalent les pauses (/), les signaux vocaux (D, E, F : heu), les faux départs (F:si que euh / y a pas grand-chose que je ieux demande ... ), les reformulations (E : si y ont des problèmes / n'importe quel problème qu'i vont avoir), les pannes lexicales (B : comme / comme; $\mathrm{D}:$ des des), et les commentaires épilinguistiques ( $\mathrm{D}:$ on appelle ça en anglais des « joint ventures»).

\subsection{La prononciation}

On aura reconnu dans les échantillons (3.1) les trucages orthographiques utilisés afin de représenter des faits de prononciation systématiques dont ne rend pas compte l'orthographe française, qui fonctionne plus sur le principe morphologique que phonétique. L'élision de consonnes touche la prononciation des pronoms ( $i l=>i$; $i l s=>i$ ou $i z$, si la liaison se fait; lui $=>y$; leur $\Rightarrow l e u$ ou $i e u x)$ et les consonnes consécutives dans le mot (autre $\Rightarrow$ aut; noucle $\Rightarrow>$ nouc; piastre $=>$ piasse; j'suis $=>$ chus; [ne] plus=>pus). L'élision d'une voyelle s'applique lorsque deux voyelles se rencontrent dans le mot, mais elle opère différemment selon qu'il s'agit d'un verbe (je suis $=>$ chus - élision de la $2^{\mathrm{e}}$ voyelle) ou d'un mot grammatical ( $p u i s=>p i s$, bien $=>b e n-$ élision de la $1^{\text {re }}$ voyelle). Ces procédés se produisent dans toutes les variétés de français familier ou populaire, ils suivent les tendances de la mécanique articulatoire.

Il n'y a donc rien de typiquement acadien, ni de canadien, dans les procédés que nous venons d'énumérer. Du point de vue extralinguistique, il y a lieu de se demander s'ils sont plus marqués socialement (stigmatisés) dans les français hexagonaux que dans les français nord-américains.

\section{Restructurations intrasystémiques}

\subsection{Morphologie}

L'envers de ce que l'on vient de voir, l'ajout de consonne, s'explique par des processus de restructuration de règles morphologiques du français. La consonne latente d'un mot (comme le «t $\mathrm{t}$ de favoriT, qui ne se prononce qu'au féminin 
favorite) est prononcée systématiquement chez beaucoup de locuteurs acadiens au «mépris» de la règle grammaticale en vigueur (A : plus $=>$ pluS; tout, toutes, tous $=>$ touT; $\mathrm{D}$ : fait/faite=>faiT). L'application de la règle morphologique s'étend à des contextes inappropriés d'après le français de référence. Il y a surgénéralisation de l'accord du participe passé féminin à des mots se terminant par le son [i], sans qu'il n'y ait de « $\mathrm{t}$ » latent (à l'écrit) : iciT, béniT, pourriT...

\subsection{Morphologie des verbes irréguliers}

La formation des verbes irréguliers (D: les personnes qu'allent de l'hôpital) pose historiquement problème. Tôt ou tard, on en vient à les conjuguer sur le modèle de verbes réguliers, soit sur un radical unique. Les exemples abondent : s'assir, on s'assisait, vous disez, vous faisez, qu'il faise. Le principe de l'analogie s'applique aussi de façon locale, dans la formation des verbes être et avoir à une seule personne et un seul temps, soit la $3^{\mathrm{e}}$ personne du pluriel de l'imparfait sontaient, ontaient et ontvaient ${ }^{11}$. Ces verbes sont attestés en acadien et dans les français de l'Ontario et de l'ouest canadien (Hallion Bres 2007 : 116). Ils apparaissent dans le parler enfantin au cours de la phase de développement morphologique puis ils disparaissent quand l'enfant réalise, grâce à l'input ambiant ou une correction explicite, qu'il applique une règle erronée. Dans les communautés minoritaires qui ont été longtemps privées de scolarisation, ces formes sont perçues comme normales, elles font maintenant partie de la grammaire de cette variété.

\subsection{Sélection de l'auxiliaire être et avoir}

Les faits indiquent qu'en acadien, l'auxiliaire avoir s'applique de façon systématique dans la formation du passé (A : l'arbre s'a ouvri; dans le corpus $\mathrm{AM}^{12}$ : L12 aussitôt j'ai arrivé chez nous; L04 une chose importante qu'a arrivé à moi) ${ }^{13}$. Ainsi, une opposition se fait dans la grammaire des français acadiens entre l'auxiliaire avoir qui dénote le passé et être, qui se spécialise comme marqueur du passif et de l'accompli. Il a mouri/mouru dénote un processus passé et il est mort, l'aspect accompli. Nous ne connaissons pas de travaux quantitatifs sur l'usage de l'auxiliaire avoir avec les verbes qui sélectionnent normalement être en français hexagonal et québécois. Cet usage a cours en Ontario et dans l'ouest canadien (Hallion Bres 2006 : 118).

\footnotetext{
${ }^{11}$ La règle $\mathrm{du}$ français est d'ajouter la flexion -aient à un seul radical par verbe (aim-/finiss-/ét-/av-) La règle dans la variété acadienne est $\mathrm{d}$ 'ajouter la flexion -aient à la forme du présent : aiment=> aimaient, finissent $=>$ finissaient, et donc sont $=>$ sontaient, ont $=>$ ontaient. Ontvaient fait la synthèse : ont+vait.

${ }^{12}$ Locuteur 12 et locutrice 04 du corpus Anna-Malenfant (Chevalier et Gauvin 1994).

${ }^{13}$ Signalons au passage, dans le dernier exemple, une illustration de la restructuration du système de pronom mentionnée au point 2.2, alinéa 2) : arrivé à moi.
} 


\subsection{Avancée du futur analytique}

La forme analytique du futur ( $\mathrm{C}: i$ vont me le donner; $\mathrm{D}: c^{\prime}$ est moi qu'i vont appeler; c'est pas moi qui va changer) est en compétition avec la forme synthétique (F: $i$ m'aideront pas avec là) depuis le XVII ${ }^{\mathrm{e}}$ siècle (Marchello-Nizia 2007). La forme analytique a plus d'avance au Québec qu'en Acadie, et plus en Acadie qu'en français hexagonal $^{14}$ (Chevalier 1996). Nous savons que le processus suit son cours en Ontario et dans l'ouest (Hallion Bres 2007: 115), mais nous n'en connaissons pas les proportions. L'explication courante de l'attirance des français d'Amérique du Nord pour la forme analytique est précisément l'analycité reconnue de l'anglais. S'agissant du futur, elle est sujette à caution, car c'est la région du N.-B. qui se trouve en situation de contact le plus intense avec l'anglais qui conserve le mieux la forme synthétique.

\subsection{Restructurations syntaxiques des subordonnées}

\subsubsection{Les interrogatives indirectes}

L'énoncé de " $\mathrm{A} »$ : il a wishé qu'i savait touT quoi-ce que les fourmis faisaient... illustre l'effet du principe de régularisation au niveau syntaxique. L'interrogation indirecte est construite sur le même modèle que l'interrogation directe (quoi-ce quelqu'est-ce que les fourmis faisaient?), ce qui réduit le nombre de règles de construction. La règle d'alternance en vigueur en français de référence, à savoir entre qu'est-ce que et ce que ne s'applique pas dans la grammaire de «A ». S'il en est de même dans les registres populaire ou familier en français hexagonal (Gadet 1999) et québécois (Tellier 2003), nous croyons par contre que l'usage du pronom fort (quoi) plutôt que faible (que) est propre aux variétés acadiennes.

\subsubsection{Les relatives}

La relative de «F»: et y a pas grand-chose qu'i m'aideront pas avec illustre la tendance à réduire le système des pronoms relatifs aux formes simples (qui et $q u e)$ et à laisser la préposition à la fin de la phrase. La construction qui en résulte est désignée sous le vocable de périphrase verbale ou de préposition orpheline ("preposition stranding» en anglais). La périphrase verbale n'est pas nécessairement associée à la relative (C: faut que je travaille pour [sous entendu : pour que mes parents me donnent de l'argent]). Il est important de souligner que dans les variétés acadiennes et laurentiennes en situation de contact intense, la

${ }^{14}$ L'usage du futur analytique est passé de $79 \%$ à $85 \%$ entre 1970 et 1984 dans le Corpus de Montréal (Émirkanian et Sankoff 1985; Zimmer1994). Dans les années 1990, il était de $92 \%$ dans la région d'Edmundston, de $78 \%$ dans la péninsule acadienne, de $62 \%$ dans le sud-est (Chevalier 1996) et de 49 \% en France (JeanJean 1988). 
construction s'étend à tout le répertoire de prépositions (qu'est-ce que tu cherches pour? qui-ce tu penses à?? qu'est-ce tu parles de? ?) plutôt que de s'en tenir aux prépositions sémantiquement pleines comme en français québécois (Golembeski 1999; Hallion Bres 2006; King 2000; Roberge 1998).

\subsubsection{Les constructions infinitives}

Il $n^{\prime} y$ a pas de constructions infinitives dans notre échantillon, mais la question mérite qu'on s'y arrête. Novale (2008) montre que les locuteurs du corpus Parkton (Moncton, N.-B., CRLA 1995) réduisent le système des prépositions et autres relateurs devant les constructions infinitives à la préposition à. Par analogie à un triple-axel est facile à réussir, on obtient il est difficile à (de) réussir un triple-axel, ii) j'ai trois jours à (pour) faire ce travail, iii) j'ai trois personnes à (à qui) téléphoner, iv) j'ai pas de place à (où) courir. On impute la confusion entre à et de dans la phrase (i) au fait qu'il n'y a que la préposition to en anglais, mais cela n'explique pas les phrases (ii) et (iii) qui sont d'un type tout à fait différent. Il faut examiner la question en élargissant les données à d'autres corpus et d'autres variétés (Chevalier, en préparation). Quelle qu'en soit la raison, il s'agit bien d'une restructuration du système des subordonnées.

\section{Changements intersystémiques : le transfert}

\subsection{Les calques}

Il y a peu d'emprunts dans les extraits en français surveillé (D, E et F.); en revanche, les calques abondent : D : voir pour des octrois (to look for "chercher", « essayer d'obtenir » des octrois); regarder à ça (to look at it, surveiller la situation, suivre les développements, ou tout simplement voir à ça, y voir); E : je fais sûr que c'est faiT (to make sure "voir à ce que », "s'assurer que cela se fasse »).

\subsection{Les emprunts lexicaux}

Les formes anglaises relevées dans nos extraits appartiennent à toutes les catégories grammaticales ou presque : a) le nom (A : un wish; $\mathrm{B}:$ bum; $\mathrm{C}:$ brats, movies; $\mathrm{E}$ : les shift); $\mathrm{b}$ ) le verbe (A : a wishé; $\mathrm{B}:$ turnont out; $\mathrm{C}:$ je babysitte), $\mathrm{d}$ ) les conjonctions (C: but, ainsi que so, well et (be)cause) et les marqueurs discursifs ( $\mathrm{C}$ : whatever, ok, anyway(s)) de même que, dans les corpus acadiens, e) des adjectifs : nice, cool, tiny, yucky, pissé off, gross, cute, frigen, fucking, f) des adverbes: right, still, though, right out, big time, about time, g) des interjections, exclamations et autres marqueurs discursifs dérivés de mots de toutes catégories, incluant les jurons : cool, gross, holy, my God, goddam, holly shit, ah well, who cares, whatever... 
Le degré d'anglicisation du vocabulaire s'évalue à la fréquence des formes anglaises en discours et à la composition du vocabulaire en langue. Plus l'emprunt touche les catégories grammaticales, plus l'influence de la langue de contact est profonde. Dans les communautés francophones majoritaires, l'emprunt touche surtout la classe des noms et des verbes et se limite à des domaines spécifiques (la mécanique, les technologies, par exemple). En milieu minoritaire, on l'a vu au paragraphe précédent, il couvre le spectre entier des champs notionnels et des classes de mots, incluant celle des conjonctions ou des prépositions et, à la limite, les pronoms et les déterminants comme en chiac de Moncton: des nice ones et avec ton own argent à moi (Perrot 2003 : 276). Les emprunts à l'anglais viennent en fait du noyau dur du lexique de la langue, soit les formes statistiquement les plus fréquentes, les plus usuelles. Ces mots viennent remplacer les mots français chez les locuteurs qui font un usage restreint $\mathrm{du}$ français parce qu'ils ne reçoivent pas un input suffisamment diversifié et continu dans leur langue pour assurer une base aussi solide qu'en anglais. Ceci explique le transfert massif vers l'anglais dans la zone des procédés expressifs : interjections, marqueurs interactifs, jurons (Chevalier et Doucet 2005).

Fréquence et saillance des mots de la langue majoritaire expliquent également l'emprunt dans la variété acadienne du sud-est du N.-B., d'une structure lexicale très productive en anglais pourtant étrangère aux langues romanes, celle des verbes à particule : B : j'veux pas que mes enfants turnont out comme s'être des bums ${ }^{15}$ (Perrot 1995; Chevalier et Long 2005; Long 2008). Pour terminer, soulignons que les éléments lexicaux empruntés dans les parlers minoritaires sont intégrés à peu près intégralement et conformément aux règles de la langue source ${ }^{16}$ sans transgresser les règles fondamentales du français.

\subsection{Le transfert codique}

Nous n'avons pas inclus les mots joint ventures, private, public partnership (D) dans la liste des emprunts, plus haut (7.2), pour la simple raison que dans ce contexte le locuteur fait du transfert codique, appelé également «alternance de code ». Le locuteur « $\mathrm{D}$ » passe du français à l'anglais et vice versa dans la phrase que nous reprenons en partie, parce qu'il y a panne lexicale : la terminologie française ne

\footnotetext{
${ }^{15}$ Avant la thèse de Golembeski sur le français du Nord de l'Ontario, cette structure a reçu peu d'attention dans les travaux sur la variation. Une locutrice originaire de Hearst confirme que les périphrases verbales (penser à; être fier de) et les verbes à particules y sont courants.

${ }^{16}$ Chevalier et Hudson (2005) montre à quel point les jeunes Québécois utilisent sans vergogne adverbe intensifieur full à toutes les sauces, sauf anglaise, alors que right en acadien, est incorporé intégralement de l'anglais régional vernaculaire.
} 
vient pas: D : des des / on appelle ça en anglais des joint ventures avec le private euh and public partnership alors ça...

Décortiquons cette séquence. Après un moment d'hésitation, de recherche des termes français pour désigner certaines opérations, « $\mathrm{D}$ » signale par un commentaire épilinguistique on appelle ça en anglais des... qu'il doit nommer les choses dans cette autre langue. Notons que le déterminant est français, mais que l'accord du nom suit la règle anglaise (sinon, on aurait omis le « $\mathrm{s} »)$. « $\mathrm{D}$ » tente de poursuivre en français avec le, puis il revient à l'anglais avec private, dernière tentative infructueuse de revenir au français euh, puis capitulation jusqu'à la fin de la phrase and public partnership. Le locuteur ré-embraye en régime français avec le marqueur alors, et le pronom résomptif ça, récupérant du coup le topo anglais dans un discours français, sans avoir à le nommer.

\section{Conclusion}

Notre exploitation des extraits s'arrête ici pour passer à la conclusion ${ }^{17}$. On aura peut-être décelé une intention didactique derrière notre exposé des particularismes dans les français du Canada, celle de ne pas conclure péremptoirement à l'anglicisme dès qu'une forme ou une expression s'écartent $\mathrm{du}$ français de référence ${ }^{18}$. La notion d'anglicisme peut, à la rigueur, être utile pour situer un phénomène, mais le danger d'y recourir trop aisément, est que cette notion nous empêche d'approfondir notre compréhension des variétés et de se débarrasser des perceptions distordues qui déteignent ensuite sur les individus et les communautés minoritaires.

Face à un usage douteux, il y a lieu de se demander si la forme n'est pas un reliquat des usages des pionnières $(\mathrm{sic})^{19}$ ou une innovation postérieure à la colonisation qui suivrait une tendance naturelle du français. Si l'analyse mène à la conclusion qu'il s'agit d'un transfert linguistique, il convient alors de se poser les questions suivantes: pourquoi emprunter, qui le fait, dans quelles circonstances, qu'est-ce qu'on emprunte, qu'est-ce qu'on n'emprunte pas, qu'est-

\footnotetext{
${ }^{17}$ Les lecteurs pourront à loisir exercer leur raisonnement linguistique en tentant d'expliquer les écarts vis-à-vis du français de référence dans les extraits B : finir s'être des bums; C : pantoute; avoir cinq piasses; chez nous; faut je travaille; E : d'aut'contact, d'aut' gens; pour / la soirée / pour la nuit disons; dépendant les shif(ts).

${ }^{18}$ On soupçonne cent ans passés, courant en Acadie, d'être un calque pour la simple raison qu'on y voit un parallèle avec hundred years ago. On ne flaire pas d'anglicisme dans la parallélisme entre sur la table et on the table). Même s'il y a eu emprunt dans les deux sens, à différentes époques, les similitudes entre le français et l'anglais peuvent être dues à la grammaire de deux langues cousines.

${ }^{19} \mathrm{~J}^{\prime} u t i l i s e$ le féminin puisque c'est surtout à elles que l'on doit la perpétuation de la langue.
} 
ce qu'on retient de la chose empruntée, comment s'intègre-t-elle dans notre langue?

Le croisement de la théorie des souches et de celle du contact des langues aide à expliquer les points de divergence entre les français acadiens et laurentiens en retraçant leur filiation aux parlers français transplantés, dans le premier cas, et, dans le second cas, à expliquer les points de convergence entre les parlers acadiens du sud-est du N.-B. et ceux de l'Ontario et de l'ouest. Nous avons fait la part entre les faits qui s'expliquent par le système français (intrasystémiques) et ceux qui sont attribuables à l'influence de l'anglais (intersystémiques). Bien entendu, la dynamique entre les deux systèmes est aussi largement influencée par les faits de société (extra-systémiques). Leur influence façonne les langues, mais les résultats sont fonction des systèmes en jeu.

Notre objectif était, plutôt que de faire un inventaire complet des particularismes des parlers acadiens et laurentiens, de donner un cadre de réflexion pour aborder les faits de langue dans les communautés linguistiques périphériques. Nous fournissons par la même occasion un inventaire d'ouvrages de consultation généraux (qui ne sont pas nécessairement cités dans l'article) et des références à des travaux ponctuels, en attendant la parution, souhaitable, d'une synthèse des travaux sur les variétés du français du Canada et leur dynamique interne et externe. Nous espérons enfin avoir engagé un dialogue à cultiver entre la linguistique descriptive, le variationnisme et la traductologie. 
http://ejournals.library.ualberta.ca/index.php/af

\section{RÉFÉRENCES BIBLIOGRAPHIQUES}

BARONIAN, Luc (2006), «Les français : état des lieux, états de la recherche, perspectives futures ", Revue Canadienne de Linguistique Appliquée/Canadian Journal of Applied Linguistics 9.2 et la Revue de l'Université de Moncton 37.2, 9-20.

BEAULIEU, Louise (1996), "Qui se ressemble s'assemble et à s'assembler on finit par se ressembler : une analyse sociolinguistique de la variable si / si que en français acadien du nord-est du Nouveau-Brunswick » dans Lise Dubois et Annette Boudreau (dirs.), Les Acadiens et leur(s) langue(s) : quand le français est minoritaire, Moncton, Éditions d'Acadie, 91-111.

BEAULIEU, Louise et Wadyslaw CICHOCKI (2005), « Facteurs internes dans deux changements linguistiques affectant l'accord sujet-verbe dans une variété de français acadien » dans Patrice Brasseur et Anika Falkert (dirs.), Français d'Amérique : approches morphosyntaxiques, Paris, L'Harmattan, 171-186.

BRASSEUR, Patrice et Anika FALKERT, dirs. (2005), Français d'Amérique : approches morphosyntaxiques, Paris, Éditions l'Harmattan.

BRASSEUR, Patrice (2001), Dictionnaire des régionalismes du français de TerreNeuve, Tübingen, Niemeyer.

BRASSEUR, Patrice (1998), Français d'Amérique: Variation, créolisation, normalisation dans Actes du colloque «Les français d'Amérique du Nord en situation minoritaire » tenu à l'Université d'Avignon, 8-11 octobre 1996, Avignon, Centre d'études canadiennes d'Avignon et du Vaucluse.

CHAUDENSON, Robert, Raymond MOUGEON et Édouard BENIAK (1993), Vers une approche panlectale de la variation du français, Paris, Didier Érudition.

CHEVALIER, Gisèle (2006), Corpus de la Baie Sainte-Anne. La reformulation d'un conte par des enfants de 4 à 10 ans, Moncton, Université de Moncton.

CHEVALIER, Gisèle (1996), «L'emploi des formes du futur dans le parler acadien du sud-est du Nouveau-Brunswick » dans Lise Dubois et Annette Boudreau (dirs.), Les Acadiens et leur(s) langue(s) : quand le français est minoritaire, Moncton, Éditions d'Acadie, 75-89.

CHEVALIER, Gisèle et Michael LONG (2007), « Les flexions acadiennes et leur formalisation pour le traitement automatique » dans Actes du $28^{\mathrm{e}}$ colloque de $l^{\prime}$ Association de linguistique des provinces Atlantiques tenu à Orono (Maine) du 3-5 novembre 2004, Orono, University of Maine, 5-14.

CHEVALIER, Gisèle et Bernise DOUCET (2005), « S'exclamer en chiac : du français ou de l'anglais? ? dans Jean Morency et al. (dirs.), Des cultures en contact : vision de l'Amérique du Nord francophone, Québec, Éditions Nota Bene, 265-281.

CHEVALIER, Gisèle et Chantal HUDSON (2005), « Deux cousins en français d'Amérique : right et full » dans Patrice Brasseur et Anika Falkert (dirs.), 
Français d'Amérique : approches morphosyntaxiques, Paris, Éditions l'Harmattan, 272-284.

CHEVALIER, Gisèle et Michael LONG (2005), « Finder out, pour qu' on les frig pas up, comment c'qui workont out, les verbes à particules en chiac » dans Patrice Brasseur et Anika Falkert (dirs.), Français d'Amérique : approches morphosyntaxiques, Paris, Éditions 1'Harmattan, 201-212.

CHEVALIER, Gisèle, Sylvia KASPARIAN et Max SILBERZTEIN (2004), «Éléments de solution pour le traitement automatique d'un français oral régional », TAL, Traitement automatique du langage 45.2, 41-62.

CHEVALIER, Gisèle et coll. (2004), Glossaire de la santé. [En ligne] (www.cafnb.ca $>>$ ressources et aide en français $>>$ parlers régionaux $>>$ glossaire acadien de la santé.)

CHEVALIER, Gisèle et Karine GAUVIN (1994), Corpus Anna-Malenfant. Enquêtes auprès d'adolescents $d u$ sud-est du Nouveau-Brunswick, Moncton, Université de Moncton.

CORMIER, Yves (1999), Dictionnaire du français acadien, Saint-Laurent, Fidès. CRLA (1995), Corpus Parkton, Moncton, Centre de recherche en linguistique appliquée, Université de Moncton.

ÉMIRIKANIAN, Louisette et David SANKOFF (1985), « Le futur simple et le futur périphrastique » dans Monique Lemieux et Henrietta J. Cedergren (dirs.), Les tendances dynamiques du français parlé à Montréal, Québec, Office de la langue française, T.1, 189-204.

FLIKEID, Karine (1989a), « Recherches sociolinguistiques sur les parlers acadiens du Nouveau-Brunswick et de la Nouvelle-Écosse » dans Raymond Mougeon et Édouard Beniak (dirs.), Le français canadien parlé hors Québec, Québec, Les Presses de l'Université Laval, 183-199.

FLIKEID, Karine (1989b), « Moitié anglais, moitié français. Emprunts et alternance de langues dans les communautés acadiennes de la Nouvelle-Écosse », Revue québécoise de linguistique théorique et appliquée 8.2, 177-228.

GADET, Françoise (1999), Le français ordinaire, Paris, Armand Colin.

GOLEMBESKI, Dan (1999), French Language Maintenance in Ontario: A

Sociolinguistic Portrait of Hearst. Thèse de doctorat inédite, Indiana University.

HALLION BRES, Sandrine (2006), « Similarités morphosyntaxiques des parlers français de l'Ouest canadien ", Revue Canadienne de Linguistique Appliquée/Canadian Journal of Applied Linguistics 9.2 et la Revue de l'Université de Moncton 37.2, 99-111.

JEANJEAN, Colette (1988), « Le futur simple et le futur périphrastique en français parlé. Étude distributionnelle » dans Claire Blanche-Benveniste, 
André Cherval et Maurice Gross (dirs.), Grammaire et histoire de la grammaire, Hommage à la mémoire de Jean Stefanini, Aix-en-Provence, Presses de l'Université de Provence, 235-257.

KING, Ruth (2000), The Lexical Basis of Grammatical Borrowing. A Prince Edward Island French Case Study, Amsterdam, Benjamins.

LONG, Michael (2008), Les verbes à particule (Vpart) dans une variété de français acadien, le chiac. Thèse de maîtrise inédite. Université de Moncton.

MARCHELLO-NIZIA, Christiane (2007), La grammaticalisation, Louvain-la-neuve, Duculot.

MOUGEON, Raymond (2005), "Postface », Cahiers franco-canadiens de l'Ouest 16.1-2, Winnipeg, Presses universitaires de Saint-Boniface, 225-239.

MOUGEON, Raymond (2000), « Les emprunts au vocabulaire de base de l'anglais en français ontarien » dans Danièle Lantin et Claude Poirier (dirs.), Contacts de langues et identités culturelles, Québec, Les Presses de l'Université Laval, 29-45.

MOUGEON, Raymond et Édouard BENIAK, dirs. (1989), Le français canadien parlé hors Québec. Aperçu sociolinguistique, Québec, Les Presses de l'Université Laval.

NOVALE, Anastassia (2008), Les particularités d'emploi des prépositions «à » et "de » suivies de la construction infinitive dans le français acadien. Exemple du corpus Parkton. Thèse de maîtrise inédite, Université de Moncton.

PAPEN, Robert A. et Gisèle CHEVALIER, dirs. (2006), Numéro thématique : «Les variétés de français parlées en Amérique. Évolution, innovation et description ", Revue Canadienne de Linguistique Appliquée/Canadian Journal of Applied Linguistics 9.2 et la Revue de l'Université de Moncton 37.2.

PÉRONNET, Louise et Sylvia KASPARIAN (2002), « Description du processus de standardisation d'une langue régionale: le cas de la préposition de dans le français standard acadien » dans Actes $d u 25^{e}$ colloque de l'Association de linguistique des provinces atlantiques tenu à Halifax en novembre 2001, Halifax, Université Dalhousie, 109-118.

PÉRONNET, Louise et Sylvia KASPARIAN (2004), « Variation dans la langue standard : le cas du français acadien. L'exemple de la préposition à », Langue et société 42, 73-83.

PÉRONNET, Louise et Sylvia KASPARIAN (1995). Corpus Péronnet-Kasparian. Moncton, Université de Moncton.

PERROT, Marie-Ève (2003), « Le français acadien en contact avec l'anglais : analyse de situations distinctes » dans André Magord (dir.), L'Acadie plurielle. Dynamiques identitaires collectives et développement au sein des réalités acadiennes, Moncton, Centre d'études acadiennes, 267-279. 
PERROT, Marie-Ève (1995), Aspects fondamentaux du métissage français-anglais dans le chiac de Moncton (N.-B.). Thèse de doctorat inédite soutenue à l'Université de la Sorbonne Nouvelle Paris III, U.F.R. du Monde anglophone, 1995.

POIRIER, Claude (1978), «L'anglicisme au Québec et l'héritage français » dans Travaux de linguistique québécoise, Québec, Presses de l'Université Laval, 43106.

ROBERGE, Yves (1998), « Les prépositions orphelines dans diverses variétés de français d'Amérique du Nord » dans Patrice Brasseur (dir.), Français d'Amérique : Variation, créolisation, normalisation, Actes du colloque : « Les français d'Amérique du Nord en situation minoritaire ", tenu à l'Université d'Avignon, 8-11 octobre 1996, Avignon, Centre d'études canadiennes de l'Université d'Avignon et Vaucluse, 49-60.

SILBERZTEIN, Max (2008), NooJ V2, A Llinguistic Development Environement, $1^{\text {re }}$ version 2002. http://www.nooj4nlp.net/

TELLIER, Christine (2003), Éléments de syntaxe du français. Méthode d'analyse grammaire générative, Montréal, Gaëtan Morin [2e édition], 125-127.

ZIMMER, D. (1994), «'Ça va tu marcher, ça marchera tu pas, je le sais pas'. Le futur simple et le futur périphrastique dans le français parlé à Montréal », Langues et Linguistique 20, 213-226. 